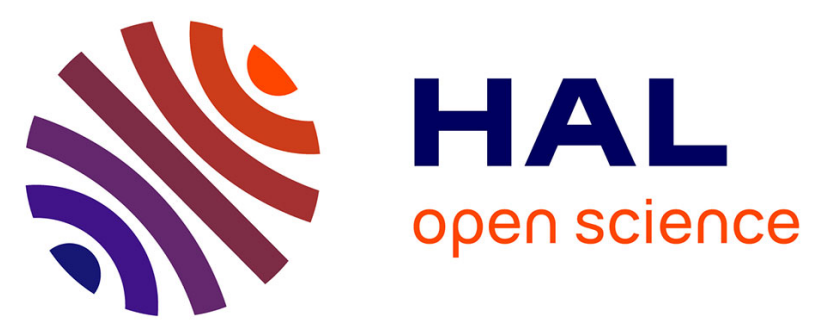

\title{
A Fast Reference Governor for the Constrained Control of Linear Discrete-time Systems with Parametric Uncertainties
}

\author{
Laurent Burlion, Marco M. Nicotra, Ilya V. Kolmanovsky
}

\section{To cite this version:}

Laurent Burlion, Marco M. Nicotra, Ilya V. Kolmanovsky. A Fast Reference Governor for the Constrained Control of Linear Discrete-time Systems with Parametric Uncertainties. 2018 IEEE Conference on Decision and Control (CDC), Dec 2018, MIAMI BEACH, United States. 10.1109/CDC.2018.8618942 . hal-02338903

\section{HAL Id: hal-02338903 https://hal.science/hal-02338903}

Submitted on 2 Nov 2021

HAL is a multi-disciplinary open access archive for the deposit and dissemination of scientific research documents, whether they are published or not. The documents may come from teaching and research institutions in France or abroad, or from public or private research centers.
L'archive ouverte pluridisciplinaire HAL, est destinée au dépôt et à la diffusion de documents scientifiques de niveau recherche, publiés ou non, émanant des établissements d'enseignement et de recherche français ou étrangers, des laboratoires publics ou privés. 


\section{A Fast Reference Governor for the Constrained Control of Linear Discrete-time Systems with Parametric Uncertainties}

\author{
Laurent Burlion
}

Marco M. Nicotra

\author{
Ilya V. Kolmanovsky
}

\begin{abstract}
This paper presents a novel reference governor scheme capable of ensuring constraint satisfaction for discretetime linear systems subject to parametric uncertainties. Given a pre-stabilized system, the proposed method generates a sequence of recursively feasible references that guarantee constraint enforcement by exploiting invariance properties. At each time step, it is shown that the next reference can be computed in closed-form by solving a set of simple second order inequalities. Parametric uncertainties are then addressed by either computing a quadratic common Lyapunov function or using suitable bounds on the parameter-dependent Lyapunov function. The efficiency of the method is illustrated by means of numerical examples.
\end{abstract}

Index Terms- uncertain systems, constrained systems, optimal control, reference governors.

\section{INTRODUCTION}

A common issue in control engineering is the discrepancy between the nominal model of a real-world plant and its actual dynamic behavior. Parametric uncertainties can be particularly problematic in the presence of system constraints, since the unforeseen mismatch between the nominal model and the plant behavior may lead to undesired constraint violations. As a result, one of the major challenges in constrained control is the need to guarantee constraint enforcement even in the presence of uncertain parameters.

Constrained control under parametric uncertainties has been addressed in the context of Model Predictive Control (MPC). Possible solutions include generating a control action that takes into account all possible trajectories of the uncertain system [1], using a local controller to confine the effects of model uncertainties to a tube centered on a nominal trajectory [2]-[4], and computing a sequence of one-step controllable ellipsoidal sets [5], [6].

Another class of solutions for constrained systems is the Reference Governor (RG), which is an add-on unit capable of ensuring constraint satisfaction by manipulating the reference of a pre-stabilized system [7], [8]. Robust reference governor schemes have been developed using both trajectory prediction tubes [9]-[11] and invariance-based considerations [12], [13]. Applications have been reported in [14], [15].

L. Burlion is with the Department of Information Processing and Systems, ONERA - The French Aerospace Lab, Toulouse, France. His sabbatical at the University of Michigan is partly funded by DGA/MRIS. lburlioneonera.fr.

M.M. Nicotra is with the University of Colorado Boulder, CO 80309. He was previously affiliated with the University of Michigan where his work was supported by the NSF award number CMMI-1562209. marco.nicotraecolorado.edu.

I.V. Kolmanovsky is with the University of Michigan, Ann Arbor, MI 48109. His work is supported by the NSF award CMMI-1562209. ilyaeumich.edu.
As detailed in the recent survey [8], most RG formulations generate an online optimization problem that is typically less computationally expensive to solve than more general MPC methods. A closed-form solver, referred to as a "Fast RG" (FaRG), has been presented in [16] for the case of linear time-invariant systems. Moreover, the recently introduced Explicit Reference Governor (ERG) has shown that it is possible to avoid solving the optimization problem altogether by manipulating the reference in a continuous-time setting [17]. A digital implementation of the ERG has been reported in [18], and relies on the assumption that the sampling period is sufficiently small to ensure the accuracy of forward Euler approximations.

The objective of this paper is to develop a Fast RG scheme for the constrained control of linear systems with parametric uncertainties. The contribution of the paper is twofold: first, it illustrates how the tools developed in the ERG framework can be tailored to discrete-time to obtain an optimization problem that admits a closed-form solution; second, it provides a systematic off-line approach for constructing robust strongly invariant sets that guarantee constraint enforcement for any parameter belonging to a convex polytope.

The paper is organized as follows. Section II introduces a novel discrete-time ERG scheme for the case of perfectly known model parameters, where the system trajectories can be bounded using Lyapunov level-sets. Section III extends this result to the case of uncertain parameters by either i) employing a Quadratic Common Lyapunov Function (QCLF), or ii) constructing suitable bounds based on a Parameter Dependent Lyapunov Function (PDLF). Numerical comparisons with existing schemes are then presented in Section IV. Section V concludes the paper with a brief summary and some closing remarks.

\section{DiSCRETE-TIME IMPLEMENTATION OF THE EXPLICIT REFERENCE GOVERNOR FOR LINEAR SYSTEMS}

The objective of this section is to illustrate how the typically continuous-time ERG framework can be tailored to the discrete-time case. To this end, we consider a discretetime linear time invariant model in the form

$$
\begin{aligned}
x^{+} & =A x+B v, \\
y & =C x+D v,
\end{aligned}
$$

where $x^{+}$denotes the successor of the plant state $x \in \mathbb{R}^{n_{x}}$, $v \in \mathbb{R}^{n_{v}}$ is the reference input, and $y \in \mathbb{R}^{n_{y}}$ is the constrained output vector which must satisfy

$$
y \in \mathcal{Y}:=\{y \mid y \leq h\} .
$$


For the remainder of the paper $C_{i}$ (resp. $D_{i}$ ) will be used to indicate the $i^{t h}$ row of matrix $C$ (resp. D) for $i \in\left\{1: n_{y}\right\}$. The components of $h$ are designated by $h_{i}$.

Assumption 1: $\left\|C_{i}\right\|>0, \forall i \in\left\{1: n_{y}\right\}$.

\section{Assumption 2: $A$ is a Schur matrix.}

Note that Assumption 2 is reasonable given that reference governors are used to augment a pre-stabilized closed-loop system. Following from this assumption, $I-A$ is invertible and the associated steady state $\bar{x}_{v}$ satisfies

$$
\bar{x}_{v}=\bar{C} v
$$

with $\bar{C}=(I-A)^{-1} B v$. Based on existing ERG results, the following subsection details how constraint enforcement can be ensured using Lyapunov functions.

\section{A. Lyapunov-based ERG}

Consider a family of Lyapunov functions of the form

$$
V_{i}(x, v)=\left(x-\bar{x}_{v}\right)^{T} P_{i}\left(x-\bar{x}_{v}\right),
$$

where the matrices $P_{i}$ are computed $\forall i \in\left\{1: n_{y}\right\}$ by solving the following set of convex optimization problems offline:

$$
\left\{\begin{array}{cl}
\min & \operatorname{Trace}\left(P_{i}\right) \\
\text { s.t. } & A^{T} P_{i} A-P_{i} \leq 0 \\
& P_{i} \geq C_{i}^{T} C_{i} \\
& P_{i}>0 \\
& P_{i}=P_{i}^{T} .
\end{array}\right.
$$

Remark 1: It is worth noting that, although the condition $P_{i} \geq C_{i}^{T} C_{i}$ is not necessary for defining a Lyapunov function, it has been shown to enhance the ERG performance by aligning the $i^{\text {th }}$ Lyapunov level sets to the corresponding constraint [19]. This remark also applies to equations (19) and (23) in the next section, where the constraint $P_{i} \geq C_{i}^{T} C_{i}$ can be dropped at the expense of the FaRG performances.

Given the individual Lyapunov functions (4), let $\Gamma_{i}(v)$ be the value of the largest Lyapunov level-set that does not violate the $i^{\text {th }}$ constraint. As detailed in [19], for linear systems and quadratic Lyapunov functions these threshold values can be computed analytically as

$$
\Gamma_{i}(v)=\frac{\left(C_{i} \bar{x}_{v}+D_{i} v-h_{i}\right)^{2}}{C_{i} P_{i}^{-1} C_{i}^{T}},
$$

which, for simplicity of notation, will be rewritten using

$$
\Gamma_{i}(v)=\gamma_{i}\left(\bar{C}_{i} v-h_{i}\right)^{2},
$$

where $\bar{H}_{i}=C_{i} \bar{C}+D_{i}$ is a nonzero vector and $\gamma_{i}=$ $\left(C_{i} P_{i}^{-1} C_{i}^{T}\right)^{-1}$ is guaranteed to be a positive scalar due to Assumption 1 and equation (5). As detailed in [17], it follows from the contractive properties of Lyapunov functions that constraint satisfaction can be guaranteed by enforcing $V_{i}(x, v) \leq \Gamma_{i}(v), \forall i \in\left\{1: n_{y}\right\}$ at each time instant. To do so, it is convenient to define the dynamic safety margin

$$
\Delta(x, v)=\min _{i \in\left\{1: n_{y}\right\}} \Delta_{i}(x, v)
$$

where

$$
\Delta_{i}(x, v)=\Gamma_{i}(v)-V_{i}(x, v)
$$

The key idea of the ERG is to generate the reference $v$ in a way that recursively ensures $\Delta(x, v) \geq 0$. In previous ERG designs, this was achieved in continuous-time by manipulating the derivative $\dot{v}$ [17]. The following section illustrates how the Lyapunov-based ERG results can instead be implemented in a discrete-time setting.

\section{B. Fast implementation in discrete-time}

To compute the reference at the next timestep, consider the linear interpolation

$$
v^{+}=v+\lambda(r-v),
$$

where $v \in \mathbb{R}^{n_{v}}$ is the currently applied reference, $r \in \mathbb{R}^{n_{v}}$ is the desired reference, and $\lambda \in[0,1]$. Based on the notion of recursive feasibility [8], the following is assumed:

Assumption 3: The initial condition $(x(0), v(0))$ is such that $\Delta(x(0), v(0)) \geq 0$.

The objective of the FaRG is to maximize $\lambda \in[0,1]$ such that $\Delta\left(x, v^{+}\right) \geq 0$. This will recursively enforce Assumption 3 for all $(x, v)$ since $\Delta\left(x, v^{+}\right) \geq 0$ implies $\Delta\left(x^{+}, v^{+}\right) \geq 0$ due to the non-increasing property of Lyapunov functions. Following from equation (8), the dynamic safety margin is guaranteed to be non-negative if each of the individual quantities

$$
\Delta_{i}\left(x, v^{+}\right)=\gamma_{i}\left(\bar{H}_{i} v^{+}-h_{i}\right)^{2}-\left(x-\bar{C} v^{+}\right)^{T} P_{i}\left(x-\bar{C} v^{+}\right),
$$

is non-negative. By substituting (10), one obtains

$$
\Delta_{i}\left(x, v^{+}\right)=a_{i} \lambda^{2}+b_{i} \lambda+c_{i}
$$

with

$$
\begin{aligned}
a_{i} & =\gamma_{i}\left(\bar{H}_{i}(r-v)\right)^{2}-(r-v)^{T} \bar{C}^{T} P_{i} \bar{C}(r-v), \\
b_{i} & =2 \gamma_{i}\left(\bar{H}_{i} v-h_{i}\right) \bar{H}_{i}(r-v)+2(r-v)^{T} \bar{C}^{T} P_{i}(x-\bar{C} v), \\
c_{i} & =\Delta_{i}(x, v) .
\end{aligned}
$$

Note that, due to Assumption $3, \lambda=0$ is always a feasible solution. The scalar $\lambda$ can thus be obtained by solving the optimization problem

$$
\left\{\begin{array}{cl}
\max & \lambda \\
\text { s.t. } & a_{i} \lambda^{2}+b_{i} \lambda+c_{i} \geq 0, \quad \forall i \in\left\{1: n_{y}\right\} \\
& \lambda \leq 1 .
\end{array}\right.
$$

The following theorem provides a systematic method for computing the solution to (12) using a closed-form approach.

Theorem 1: Consider the optimization problem (12) satisfying $c_{i} \geq 0, \forall i \in\left\{1: n_{y}\right\}$, and let $I_{0}=\left\{i \mid a_{i}=\right.$ $\left.0, b_{i}+c_{i}<0\right\}$ and $I_{1}=\left\{i \mid a_{i} \neq 0, a_{i}+b_{i}+c_{i}<0\right\}$. Given the initial solution estimate

$$
\bar{\lambda}_{1}=\min \left(\lambda_{0}, \lambda_{1}, 1\right)
$$

with

$$
\lambda_{0}=\min _{i \in I_{0}} \frac{c_{i}}{-b_{i}}, \quad \lambda_{1}=\min _{i \in I_{1}} \frac{-b_{i}-\sqrt{b_{i}^{2}-4 a_{i} c_{i}}}{2 a_{i}},
$$

let the recursion counter be initialized as $k=1$ and let $I_{k+1}=\left\{i \mid a_{i}>0,-b_{i} / 2 a_{i}<\bar{\lambda}_{k}, a_{i} \bar{\lambda}_{k}^{2}+b_{i} \bar{\lambda}_{k}+c_{i}<0\right\}$. Then, the following holds 
1) If $I_{k+1}=\emptyset$, the current $\bar{\lambda}_{k}$ is the maximizer of (12);

2) If $I_{k+1} \neq \emptyset$, the estimate update

$$
\bar{\lambda}_{k+1}=\min _{i \in I_{k+1}} \frac{-b_{i}-\sqrt{b_{i}^{2}-4 a_{i} c_{i}}}{2 a_{i}},
$$

is such that $I_{k} \cap I_{j}=\emptyset, \forall k \neq j$.

Proof: Whenever $c_{i} \geq 0$ and $a_{i} \bar{\lambda}^{2}+b_{i} \bar{\lambda}+c_{i} \geq 0$, it is trivial to show that there exists $\lambda \in(0, \bar{\lambda})$ such that $a_{i} \lambda^{2}+b_{i} \lambda+c_{i}<0$ only if $a_{i}>0$ and $-b_{i} / 2 a_{i} \in(0, \bar{\lambda})$. As a result, $\forall i \in I_{k}$, the condition $a_{i} \lambda^{2}+b_{i} \lambda+c_{i} \geq 0$ is guaranteed to hold $\forall \lambda \in\left[0, \bar{\lambda}_{k}\right]$. This recursively ensures $I_{k} \cap I_{j}=\emptyset$ since $\bar{\lambda}_{j}<\bar{\lambda}_{k}, \forall j>k$. To prove that $I_{k}=\emptyset$ implies that $\bar{\lambda}_{k}$ is the solution to (12), it is sufficient to note that, by construction, $\forall \lambda \in\left(\bar{\lambda}_{k}, 1\right]$ there exists $i \in I_{0} \cup I_{1} \cup$ $\ldots \cup I_{k-1}$ such that $a_{i} \lambda^{2}+b_{i} \lambda+c_{i}<0$.

The main advantage of Theorem 1 is that it recursively guarantees constraint enforcement for all indices belonging to the set $\left\{i \mid a_{i} \leq 0\right\} \cup I_{1} \cup \ldots \cup I_{k-1}$. Consequently, $v^{+}$ is guaranteed to be assigned after solving at most $n_{y}$ first or second order equations. The following section illustrates how this result can be extended to account for model parametric uncertainties.

\section{EXTENSION TO LINEAR SYSTEMS WITH PARAMETRIC UNCERTAINTIES}

The objective of this section is to extend the results of the previous section to the following class of constrained discrete-time linear systems with parameter uncertainties,

$$
\begin{aligned}
x^{+} & =A(p) x+B(p) v, \\
y & =C x+D v,
\end{aligned}
$$

once again subject to the output constraints (2). The uncertain systems matrices $[A(p), B(p)]$ are allowed to lie in a matrix polyhedron of the form

$$
A(p)=\sum_{j=1}^{n_{p}} p_{j} A_{j} \quad, \quad B(p)=\sum_{j=1}^{n_{p}} p_{j} B_{j},
$$

where the vector $p=\left[p_{1}, \ldots, p_{n_{p}}\right]^{T}$ belongs to the unit simplex

$$
\mathcal{P}:=\left\{p \in \mathbb{R}^{n_{p}} \mid \sum_{j=1}^{n_{p}} p_{j}=1, p_{j} \geq 0\right\} .
$$

As in the previous section, it is assumed that the system has been pre-stabilized so that it asymptotically tends to a target steady-state $\bar{x}_{v}=\bar{C} v$. For details on how to design a pre-stabilizing feedback loop for parameter dependent linear systems, the reader is referred to [20], [21].

Assumption 4: $A(p)$ is a Schur matrix, $\forall p \in \mathcal{P}$.

Assumption 5: $(I-A(p))^{-1} B(p)=\bar{C}, \forall p \in \mathcal{P}$.

Note that Assumption 5 is reasonable in many applications where the steady-state of the system does not depend on the model parameters. A notable example are mechanical systems, where the steady-state is typically achieved when the position is equal to the reference and all velocities are equal to zero. The following subsections develop Lyapunov-based FaRG strategies that are robust to parametric uncertainties.

\section{A. QCLF-based Robust FaRG}

The most straightforward option for dealing with parametric uncertainties is to determine the invariants using a Quadratic Common Lyapunov Function (QCLF). This can be done by considering the same Lyapunov functions as in (4), but computing the matrices $P_{i}, \forall i \in\left\{1: n_{y}\right\}$ by solving the following offline convex optimization problems

$$
\left\{\begin{array}{cl}
\min & \operatorname{Trace}\left(P_{i}\right) \\
\text { s.t. } & A_{j}^{T} P_{i} A_{j}-P_{i} \leq 0, \forall j \in\left\{1: n_{p}\right\}, \\
& P_{i} \geq C_{i}^{T} C_{i} \\
& P_{i}>0 \\
& P_{i}=P_{i}^{T} .
\end{array}\right.
$$

If a solution to (19) exists, then the Lyapunov-based FaRG presented in the previous section can be applied with no further modifications and can even handle time-varying parameters. This is due to the fact that (19) ensures

$$
A(p)^{T} P_{i} A(p)-P_{i} \leq 0, \forall p \in \mathcal{P} .
$$

However, depending on $A(p)$, equation (20) may not admit a solution, even though Assumption 4 holds true. To overcome this issue, the following subsection addresses the case in which it is not possible to find a QCLF.

\section{B. PDLF-based robust FaRG}

To address the case when (20) does not admit a solution, let us now consider a family of Parameter Dependent Lyapunov Functions (PDLF),

$$
V_{i}(x, v, p)=\left(x-\bar{x}_{v}\right)^{T} P_{i}(p)\left(x-\bar{x}_{v}\right),
$$

which can be constructed in the discrete-time case using the result of [21, Theorem 2]. In particular, for each output constraint $i \in\left\{1: n_{y}\right\}$, it is possible to define the matrix,

$$
P_{i}(p)=\sum_{j=1}^{n_{p}} p_{j} P_{i j},
$$

with $p \in \mathcal{P}$. Each $P_{i j}$ can then be computed by solving the following convex optimization problem,

$$
\left\{\begin{array}{cl}
\min & \operatorname{Trace}\left(P_{i j}\right) \\
\text { s.t. } & \forall j \in\left\{1: n_{p}\right\}, \\
& {\left[\begin{array}{cc}
G_{i}+G_{i}^{T}-P_{i j} & G_{i} A_{j} \\
A_{j}^{T} G_{i}^{T} & P_{i j}
\end{array}\right]>0,} \\
& P_{i j} \geq C_{i}^{T} C_{i}, \\
& P_{i j}=P_{i j}^{T} .
\end{array}\right.
$$

In analogy to Section II, given the threshold value

$$
\Gamma_{i}(v, p)=\gamma_{i}(p)\left(\bar{H}_{i} v-h_{i}\right)^{2},
$$

where $\bar{H}_{i}=C_{i} \bar{C}+D_{i}$ and $\gamma_{i}(p)=\left(C_{i} P_{i}^{-1}(p) C_{i}^{T}\right)^{-1}$, constraint satisfaction can be guaranteed by enforcing the condition $\Delta(x, v, p) \geq 0$, with

$$
\Delta(x, v, p)=\min _{i \in\left\{1: n_{y}\right\}} \Delta_{i}(x, v, p),
$$

and $\Delta_{i}(x, v, p)=\Gamma_{i}(v, p)-V_{i}(x, v, p)$. Given the linear interpolation (10), it follows once again that

$$
\Delta_{i}\left(x, v^{+}, p\right)=a_{i}(p) \lambda^{2}+b_{i}(p) \lambda+c_{i}(p),
$$


with

$$
\begin{aligned}
a_{i}(p)= & \gamma_{i}(p)\left(\bar{H}_{i}(r-v)\right)^{2}-(r-v)^{T} \bar{C}^{T} P_{i}(p) \bar{C}(r-v), \\
b_{i}(p)= & 2 \gamma_{i}(p)\left(\bar{H}_{i} v-h_{i}\right) \bar{H}_{i}(r-v) \\
& +2(r-v)^{T} \bar{C}^{T} P_{i}(p)(x-\bar{C} v), \\
c_{i}(p)= & \Delta_{i}(x, v, p) .
\end{aligned}
$$

Unlike Section II, however, equation (26) cannot be used to directly compute $\lambda \in[0,1]$ since $p$ is a vector of unknown parameters. The following theorem provides a solution to this issue.

Theorem 2: Let system (16) be subject to the output constraints (2), and let Assumptions 1, 4, and 5 be satisfied. Moreover, given an unknown parameter $p \in \mathcal{P}$, let the current state $x \in \mathbb{R}^{n_{x}}$ and applied reference $v \in \mathbb{R}^{n_{v}}$ be such that $\Delta(x, v, p) \geq 0$. Then, given

$$
\underline{\gamma}_{i}=\min _{p \in \mathcal{P}} \gamma_{i}(p)
$$

and

$$
c_{i j}=\underline{\gamma}_{i}\left(\bar{H}_{i} v-h_{i}\right)^{2}-(x-\bar{C} v)^{T} P_{i j}(x-\bar{C} v),
$$

it is possible to enforce the condition $\Delta\left(x, v^{+}, p\right) \geq 0$ by assigning $v^{+}=v+\lambda(r-v)$ and choosing $\lambda \in[0,1]$ as follows:

1) If $\exists i \in\left\{1: n_{y}\right\}, j \in\left\{1: n_{p}\right\}: c_{i j}<0$, then $\lambda=0$.

2) If $c_{i j} \geq 0, \forall i \in\left\{1: n_{y}\right\}, \forall j \in\left\{1: n_{p}\right\}$, compute $\lambda$ as the solution to

$$
\left\{\begin{array}{cl}
\max & \lambda \\
\text { s.t. } & \forall i \in\left\{1: n_{y}\right\}, \forall j \in\left\{1: n_{p}\right\}, \\
& a_{i j} \lambda^{2}+b_{i j} \lambda+c_{i j} \geq 0 \\
& \lambda \leq 1,
\end{array}\right.
$$

where

$$
\begin{aligned}
a_{i j}= & \underline{\gamma}_{i}\left(\bar{H}_{i}(r-v)\right)^{2}-(r-v)^{T} \bar{C}^{T} P_{i j} \bar{C}(r-v), \\
b_{i j}= & 2 \underline{\gamma}_{i}\left(\bar{H}_{i} v-h_{i}\right) \bar{H}_{i}(r-v) \\
& +2(r-v)^{T} \bar{C}^{T} P_{i j}(x-\bar{C} v),
\end{aligned}
$$

Proof: The two cases are addressed separately:

1) The result $\Delta\left(x, v^{+}, p\right) \geq 0$ follows directly from $\Delta(x, v, p) \geq 0$ since $\lambda=0$ implies $v^{+}=v$.

2) Following from equation (27), one has

$$
\Delta_{i}(x, v, p) \geq \underline{\gamma}_{i}\left(\bar{H}_{i} v-h_{i}\right)^{2}-V_{i}(x, v, p) .
$$

Moreover, it follows from (22) that

$$
V_{i}(x, v, p) \leq \max _{j \in\left\{1: n_{p}\right\}} V_{i}\left(x, v, e_{j}\right),
$$

where $e_{j}$ is the $j^{\text {th }}$ column vector of an identity matrix of size $n_{p}$. As a result, $\Delta\left(x, v^{+}, p\right) \geq 0$ can be enforced by ensuring

$$
\underline{\gamma}_{i}\left(\bar{H}_{i} v^{+}-h_{i}\right)^{2}-V_{i}\left(x, v^{+}, e_{j}\right) \geq 0
$$

for all constraints $i \in\left\{1: n_{y}\right\}$ and all parameter vertices $j \in\left\{1: n_{p}\right\}$. This can be done by solving (29), which always admits a solution $\lambda \in[0,1]$ since $c_{i j} \geq 0, \forall i \in\left\{1: n_{y}\right\}, \forall j \in\left\{1: n_{p}\right\}$.
Remark 2: Theorem 2 details how to take advantage of invariance properties even when the invariant set is uncertain. The idea is that the (unknown) invariant set $\{x \mid \Delta(x, v, p) \geq$ $0\}$ is guaranteed to contain the set

$$
\mathcal{S}:=\left\{x \mid \underline{\gamma}_{i}\left(\bar{H}_{i} v^{+}-h_{i}\right)^{2}-(x-\bar{C} v)^{T} P_{i j}(x-\bar{C} v) \geq 0, \forall i, j\right\},
$$

which is a strongly returnable set as defined in [12]. Indeed, even though the set $\mathcal{S}$ is not invariant per se, any trajectory initialized in $\mathcal{S}$ will be contained by the invariant set $\{x \mid \Delta(x, v, p) \geq 0\}$, therefore ensuring constraint satisfaction at all times. It is also worth noting that the statement in Theorem 2 encompasses the results detailed in Subsections II-B and III-A, and that the optimization problem (29) can be solved explicitly using the closed-form algorithm proposed in Theorem 1 which here guarantees to solve at most $n_{y} \times n_{p}$ first or second order equations.

\section{NumeriCAL EXAMPLES}

Consider the following parameter-dependent linear system,

$$
x^{+}=A_{0}(p) x+B_{0} u,
$$

where $A_{0}(p)$ will be assigned in the following subsections and $B_{0}=\left[\begin{array}{ll}0 & 1\end{array}\right]^{T}$. The system is pre-stabilized using the control input

$$
u=K x-k_{1} v,
$$

with $K=\left[\begin{array}{ll}k_{1} & k_{2}\end{array}\right]=\left[\begin{array}{ll}-0.3 & -0.1\end{array}\right]$, and is subject to the state and input constraints

$$
-\left[\begin{array}{l}
10 \\
10
\end{array}\right] \leq x \leq\left[\begin{array}{l}
10 \\
10
\end{array}\right], \quad-1 \leq u \leq 1 .
$$

The resulting pre-stabilized system can be rewritten in the form (1)-(2) using

$$
A(p)=A_{0}(p)+B_{0} K, \quad B=-k_{1} B_{0}
$$

and

$$
\begin{aligned}
C & =\left[\begin{array}{llll}
I_{2} & -I_{2} & K & -K
\end{array}\right]^{T}, \\
D & =\left[\begin{array}{llllll}
0 & 0 & 0 & 0 & -k_{1} & k_{1}
\end{array}\right]^{T}, \\
h & =\left[\begin{array}{llllll}
10 & 10 & 10 & 10 & 1 & 1
\end{array}\right]^{T} .
\end{aligned}
$$

The following examples detail the implementation of the proposed FaRG to enforce constraint satisfaction depending on the properties of $A_{0}(p)$. For comparison purpose, we will detail the results obtained using a conventional RG where the output admissible sets defined in [7] are constructed using the computationally efficient method proposed in [22], [23]. The idea behind this method is to bound the trajectory predictions using polyhedral invariant sets which are obtained by systematically removing redundant constraints.

\section{A. Example 1: 'Moderate' Uncertainty}

Consider the case

$$
A_{0}(p)=\left[\begin{array}{cc}
1 & 0.1 p_{1}+0.15 p_{2} \\
0 & 1
\end{array}\right],
$$




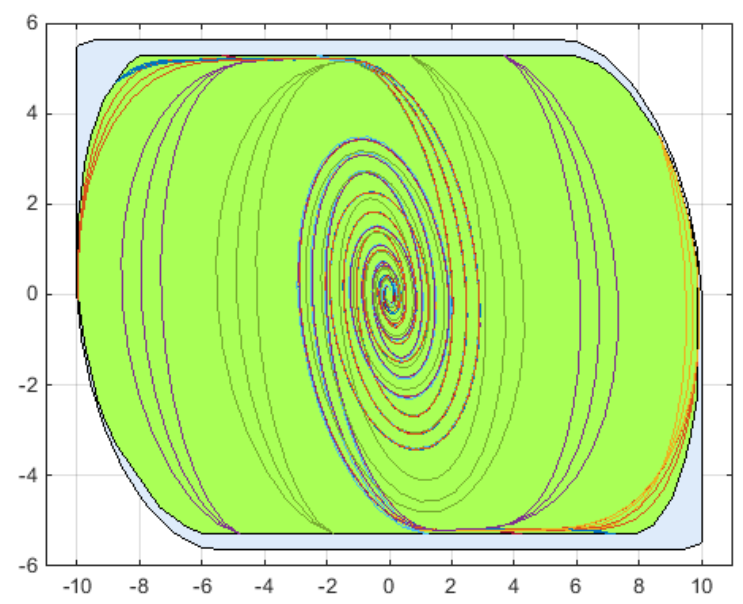

Fig. 1. Example 1: Invariant set obtained using [22, Algorithm 1C] (in blue) and invariant set obtained using the QCLF-based FaRG (in green). The system trajectories are shown to always be contained by the invariant sets.
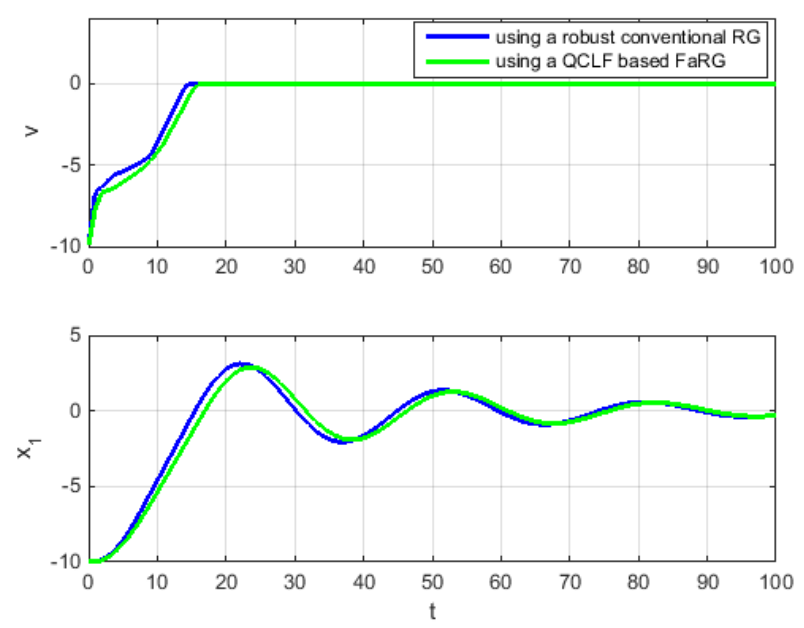

Fig. 2. Example 1: trajectories starting from $x_{0}=[-9.95 ;-0.1]$ with $v 0=-9.93, p_{1}=0, p_{2}=1$.

with $p_{1} \in[0,1]$ and $p_{2}=\left(1-p_{1}\right)$. Since the solution to (19) exists, the applied reference is updated using a QCLF-based strategy, which requires the solution of at most 6 second order equations. Comparatively, the method proposed in [22, Algorithm 1C] generates a problem with 100 non redundant constraints. Figure 1 illustrates the invariant sets obtained with the two methods, where the QCLF-based invariant (shown in green) is only slightly smaller than the invariant obtained with the conventional RG. The figure also shows that the trajectories of the closed-loop system are always contained in the invariant set. Figure 2 illustrates the output response obtained using the two methods, where it can be seen that the QCLF-based FaRG is only slightly slower than the conventional RG. This minor loss in output response performances, likely due to the smaller size of the invariant, is counterbalanced by a computational footprint that is almost 20 times smaller (6 inequality constraints versus 100).

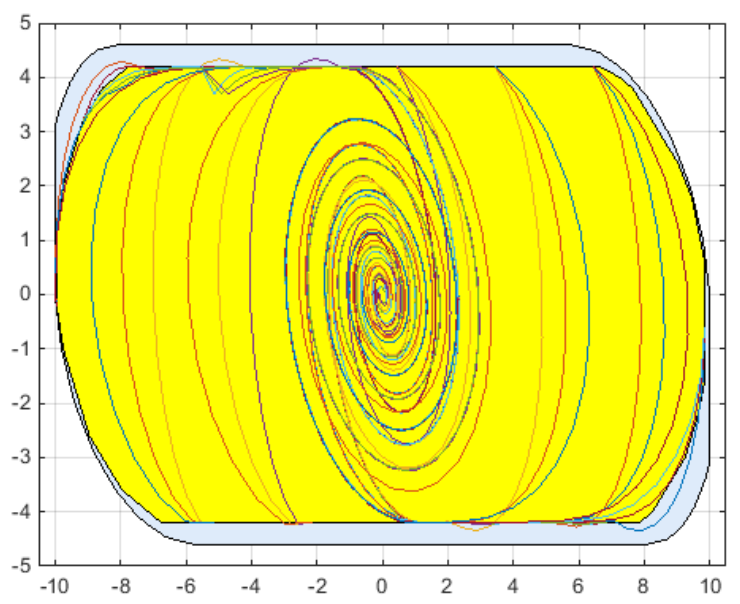

Fig. 3. Example 2: Invariant set obtained using [22, Algorithm 1C] (in blue) and strongly returnable set obtained using the PDLF-based FaRG (in yellow). The system trajectories are shown to exit the strongly returnable set, but remain bounded by the invariant.
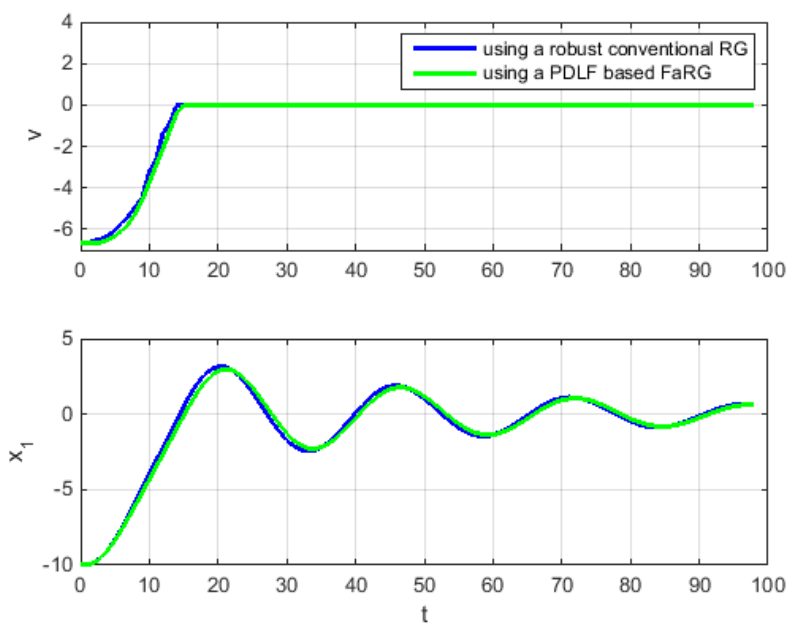

Fig. 4. Example 2: trajectories starting from $x_{0}=[-9.95 ;-0.2]$ with $v 0=-6.68, p_{1}=0, p_{2}=1$

\section{B. Example2: 'Large' Uncertainty}

Consider the case

$$
A_{0}(p)=\left[\begin{array}{cc}
1 & 0.1 p_{1}+0.2 p_{2} \\
0 & 1
\end{array}\right]
$$

where $A_{0}$ is almost the same as before except that the uncertain parameter variation is doubled. In this case, (19) does not admit a solution, whereas it is possible to solve equation (23). As a result, constraint enforcement is ensured using PDLF-based FaRG, which requires the solution of at most 12 second order equations. Comparatively, the method proposed in [22, Algorithm 1C] generates a problem with 108 non redundant inequalities. Figure 3 illustrates the invariant set obtained using the conventional RG (in blue) and the strongly returnable set employed by the PDLF-based FaRG. In this case, the system trajectories are shown to exit the strongly returnable set, but are nevertheless guaranteed to ensure 


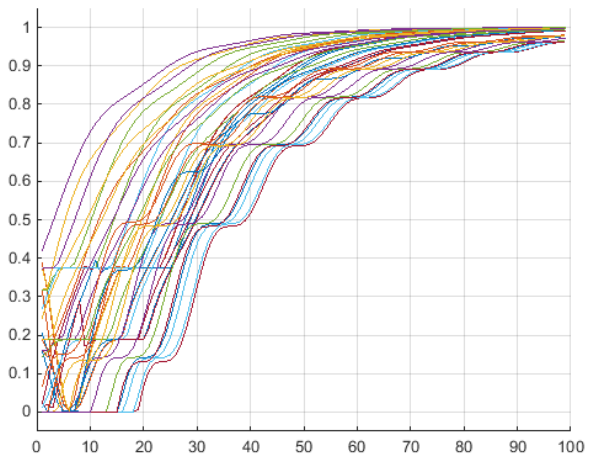

Fig. 5. Example 2: Temporal behavior of $\Delta(x, v, p)$ computed for various initial condition and parameter values. Constraint enforcement is always guaranteed due to the fact that $\Delta(x, v, p) \geq 0$

constraint satisfaction. This can be seen in Figure 5, which shows that the actual value of $\Delta(x, v, p)$ remains positive for all trajectories originating from the strongly returnable set (33). As in the previous example, Figure 4 shows that the output performance of the FaRG is comparable to the conventional RG, even though its computational burden is approximately 10 times smaller (12 vs 108).

Note that the discrepancy between the computational cost of the FaRG and the conventional RG will increase drastically for higher order systems. This is due to the fact that the complexity of the FaRG grows linearly with the system dimensions, whereas the complexity of the RG will grow geometrically.

\section{CONCLUSION}

In this paper we have developed a robust reference governor algorithm for the constrained control of uncertain linear polytopic systems. The proposed method relies on tools from the ERG framework, but is specifically formulated in a discrete-time context. The offline computational effort merely consists in determining suitable Lyapunov functions (QCLF or PDLF) for polytopic uncertain systems. The online computations are then reduced to solving an optimization problem with a small number of second order equations. Numerical results show that the proposed method can achieve performances that are comparable to robust trajectory-based approaches in terms of output response, while being significantly less computationally expensive.

\section{REFERENCES}

[1] D.R. Ramirez, T. Alamo, E.F. Camacho, and D. Muñoz de la Peña. Min-max mpc based on a computationally efficient upper bound of the worst case cost. Journal of Process Control, 16(5):511 - 519, 2006.

[2] D.Q. Mayne and E.C. Kerrigan. Tube-based nonlinear model predictive control. In Proc. of the Seventh IFAC Symposium on Nonlinear Control Systems, pages 110-115, 2007.

[3] S.V. Raković, B. Kouvaritakis, M. Cannon, C. Panos, and R. Findeisen Parameterized tube model predictive control. IEEE Transactions on Automatic Control, 57:27462761, 2012.

[4] S. Di Cairano. Indirect adaptive model predictive control for linear systems with polytopic uncertainty. In 2016 American Control Conference, pages 3570-3575, 2016.
[5] D. Angeli, A. Casavola, G. Franzè, and E. Mosca. An ellipsoidal off-line mpc scheme for uncertain polytopic discrete-time systems. Automatica, 44(12):3113 - 3119, 2008.

[6] M. Cannon, S. Li, Q. Cheng, and B. Kouvaritakis. Efficient robust output feedback mpc. In 18th IFAC World Congress, pages 79577962, 2011

[7] E. G. Gilbert and K. T. Tan. Linear systems with state and control constraints: the theory and application of maximal output admissible sets. IEEE Transactions on Automatic Control, 36(9):1008-1020, 1991

[8] E. Garone, S. Di Cairano, and I. Kolmanovsky. Reference and command governors for systems with constraints: A survey on theory and applications. Automatica, 75(Supplement C):306 - 328, 2017.

[9] A. Casavola and E. Mosca. Reference governor for constrained uncertain linear systems subject to bounded input disturbances. In Proceedings of 35th IEEE Conference on Decision and Control, volume 3, pages 3531-3536 vol.3, 1996.

[10] A. Bemporad and E. Mosca. Fulfilling hard constraints in uncertain linear systems by reference managing. Automatica, 34(4):451 - 461, 1998.

[11] A. Casavola, E. Mosca, and D. Angeli. Robust command governors for constrained linear systems. IEEE Transactions on Automatic Control, 45(11):2071-2077, 2000.

[12] E. Gilbert and I.V. Kolmanovsky. Nonlinear tracking control in the presence of state and control constraints: a generalized reference governor. Automatica, 38(12):2063-2073, 2002.

[13] M. M. Nicotra and E. Garone. An explicit reference governor for the robust constrained control of nonlinear systems. In 55th IEEE Conference on Decision and Control (CDC), pages 1502-1507, 2016.

[14] J. Sun and I. V. Kolmanovsky. Load governor for fuel cell oxygen starvation protection: A robust nonlinear reference governor approach. IEEE Transactions on Control Systems Technology, 13(6):911-920, 2005.

[15] M. M. Nicotra, R. Naldi, and E. Garone. A robust explicit reference governor for constrained control of unmanned aerial vehicles. In 2016 American Control Conference (ACC), pages 6284-6289, 2016.

[16] M.M. Nicotra, E. Garone, and I.V. Kolmanovsky. Fast reference governor for linear systems. AIAA Journal of Guidance, Control, and Dynamics, 40(2):461-465, 2017.

[17] E. Garone and M. M. Nicotra. Explicit reference governor for constrained nonlinear systems. IEEE Transactions on Automatic Control, 61(5):1379-1384, 2016.

[18] R. Romagnoli, L. D. Couto, M. M. Nicotra, M. Kinnaert, and E. Garone. Computationally-efficient constrained control of the stateof-charge of a li-ion battery cell. In 56th IEEE Annual Conference on Decision and Control (CDC), pages 1433-1439, 2017.

[19] E. Garone, M. Nicotra, and L. Ntogramatzidis. Explicit reference governor for linear systems. International Journal of Control, 0(0):116, 2017.

[20] B. Barmish. Stabilization of uncertain systems via linear control. IEEE Transactions on Automatic Control, 28(8):848-850, 1983.

[21] M.C. de Oliveira, J. Bernussou, and J.C. Geromel. A new discretetime robust stability condition. Systems \& Control Letters, 37(4):261 $-265,1999$.

[22] B. Pluymers, J. A. Rossiter, J. A. K. Suykens, and B. De Moor. The efficient computation of polyhedral invariant sets for linear systems with polytopic uncertainty. In Proceedings of the 2005 American Control Conference, pages 804-809, vol. 2, 2005.

[23] B. Pluymers, M. V. Kothare, J. A. K. Suykens, and B. De Moor Robust synthesis of constrained linear state feedback using lmis and polyhedral invariant sets. In 2006 American Control Conference, pages 6 pp.-, 2006. 